\title{
Stochastic Modeling of Repairable Redundant System Comprising One Big Unit and Three Small Dissimilar Units
}

\author{
Ibrahim Yus uf", Nafiu Huss aini \\ Department of Mathematical Sciences, Faculty of Science, Bayero University, Kano, Nigeria
}

\begin{abstract}
This paper deals with the stochastic modeling of system comprising two subsystems A and B in series. Subsystem A consists three active parallel units. Failure time and repair time are assumed exponential. We developed explicit expressions for mean time to system failure (MTSF), system availability, busy period and profit function using Kolmogorov's forward equations method and perform graphical analysis to see the behavior of failure rates and repair rates on measures of system effectiveness such MTSF, system availability and profit function.
\end{abstract}

Keywords MTSF, System Availability, Profit Function, Active Parallel

\section{Introduction}

Stochastic models of redundant systems as well as methods of evaluating system reliability indices such as mean time to system failure (MTSF), system availability, busy period of repairman, profit analysis, etc have been researched in order to improve the system effectiveness.

There are systems of three units in which two units are sufficient to perform the entire function of the system. Such systems are called 2-out-of-3 redundant systems. These systems have wide application in the real world. The communication system with three transmitters can be sited as a good example of 2-out-of-3 redundant system. Many research results have been reported on reliability of 2-out-of-3 redundant systems. For example, Chander and Bhardwaj[1], analyzed reliability models for 2-out-of-3 redundant system subject to conditional arrival time of the server. Chander and Bhardwaj[2] present reliability and economic analysis of 2-out-of-3 redundant system with priority to repair. Bhardwaj and Malik[3] studied MTSF and cost effectiveness of 2-out-of-3 cold standby system with probability of repair and inspection. Taneja el al[4] deals with the reliability and cost benefit analysis of a system consisting o a big unit and two identical small units. A single repair facility appears and disappears from the system randomly with constant rates, Malik et al[5] analyzed two reliability models for a system of non

* Corresponding author:

ibrahimyusif@yahoo.com (Ibrahim Yusuf)

Published online at http://journal.sapub.org/ajcam

Copyright (C) 2012 Scientific \& Academic Publishing. All Rights Reserved identical units original and duplicate using regenerative point technique., Mahmoud and Moshrefa[6] deal with the study of the stochastic analysis of a two unit cold standby system considering hardware failure, human error failure and preventive maintenance, Yusuf and Bala[7], studied stochastic two models of two unit parallel system. In model I, the system can be normal, deterioration (slow, mild or fast deterioration), failure whereas in model II, the system can either be in normal of failure modes. Using linear first order linear differential equations, various measures of system effectiveness such as mean time to system failure (MTSF) and availability are obtained to see the effect of deterioration on such measures, Kumar and Kadyan[8] deal with profit analysis of two unit non identical system with degradation and replacement while Sureria et al[9] studied cost benefit analysis of a computer system with priority to software replacement over hardware repair, Bhardwaj and Malik[15] developed two models for 2-out-of-3 system to study cost benefit analysis using semi-Markov and regenerative process.

\subsection{Objective}

In this paper, we study a system comprising of two subsystems A and B in series. Subsystem A consists of three active parallel units while subsystem $B$ is a single unit. The system is attended by four repairmen and considered up when: (1) all the units of subsystem $A$ and subsystem $B$ are working (2) two units of subsystem A and subsystem B are working. The system is down when two units of subsystem A failed or at the failure of subsystem B. We analyzed the system behavior using kolmogorov's forward equation methods. Explicit expression for measures of system 
effectiveness like mean time to system failure (MTSF), system availability, busy period of repairman, and profit analysis have been developed. The objective is to study the effect of failure and repair rates parameters with respect to subsystems A and B on reliability indices such as MTSF, availability and profit. Graphs were plotted to see the behavior of failure and repair rates on system performance.

\section{Notations}

$A_{i O}$ Unit $i$ in subsystem A is operational $i=1,2,3$

$A_{R i}$ Failed unit in subsystem $A$ under type $i$ repair

$A_{i G}$ Unit $i$ in subsystem $A$ is good

$B_{O}$ Subsystem $B$ is operational

$B_{R 4}$ Subsystem $B$ is failed and under type 4 repair

$\beta_{i}$ Type $i$ failure rate of unit $A_{i}$ in subsystem A

$\alpha_{i}$ Type $i$ repair rate of unit $A_{i}$ in subsystem A

$\lambda$ Failure rate of subsystem $B$

$\mu$ Repair rate of subsystem B

\subsection{Model Description and Ass umptions}

1. The system consist of two non identical subsystems A and $B$

2. Subsystem A consist three active parallel units

3. Units in subsystem A and subsystem B can have two modes: operation and failure

4. The system is attended by four repairmen

5. The system is down when two units of subsystem $A$ failed or at the failure of subsystem $B$

6. The system is up when all the units of subsystem A

and subsystem B are operational or two units of subsystem $A$ and subsystem $B$ are operational

7. Units in subsystem A suffer three types of failures while subsystem B suffer one type of failure

8. Failure rates and repair rates are constant

\subsection{State of the System}

Up states:

$$
\begin{aligned}
& S_{0}\left(A_{1 O}, A_{2 O}, A_{3 O}, B_{O}\right) \\
& S_{1}\left(A_{R 1}, A_{2 O}, A_{3 O}, B_{O}\right) \\
& S_{2}\left(A_{1 O}, A_{R 2}, A_{3 O}, B_{O}\right), \\
& S_{3}\left(A_{1 O}, A_{2 O}, A_{R 3}, B_{O}\right)
\end{aligned}
$$

Failed states:

$$
\begin{gathered}
S_{4}\left(A_{1 O}, A_{2 O}, A_{3 O}, B_{R 4}\right) \\
S_{5}\left(A_{R 1}, A_{R 2}, A_{3 G}, B_{G}\right) \\
S_{6}\left(A_{R 1}, A_{2 G}, A_{3 G}, B_{R 4}\right), \\
S_{7}\left(A_{1 G}, A_{R 2}, A_{R 3}, B_{G}\right) \\
S_{8}\left(A_{1 G}, A_{R 2}, A_{3 G}, B_{R 4}\right) \\
S_{9}\left(A_{R 1}, A_{2 G}, A_{R 3}, B_{G}\right) \\
S_{10}\left(A_{1 G}, A_{2 G}, A_{R 3}, B_{R 4}\right)
\end{gathered}
$$

\section{Model Formulation}

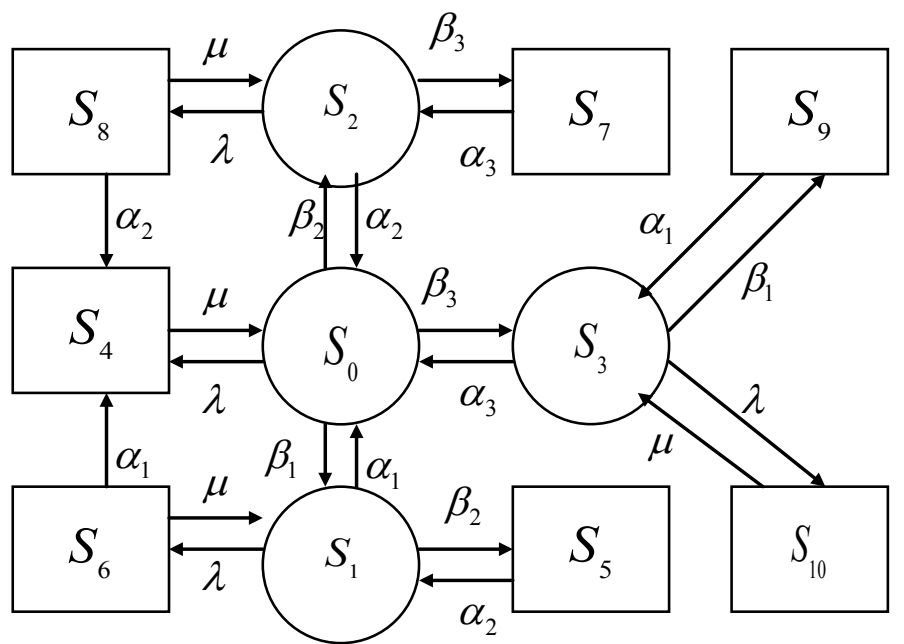

Figure 1. schematic diagram of the System

\subsection{Me an Time to System Failure for System}

Let $P(t)$ be the probability row vector at time $t$, then the initial conditions for this problem are as follows:

$P(0)=\left[P_{0}(0), P_{1}(0), P_{2}(0), P_{3}(0), P_{4}(0), P_{5}(0), P_{6}(0), P_{7}(0), P_{8}(0), P_{9}(0), P_{10}(0)\right]=[1,0,0,0,0,0,0,0,0,0,0]$ we obtain the following system of differential equations from Figure 1 above: 


$$
\begin{aligned}
& \frac{d P_{0}(t)}{d t}=-\left(\lambda+\beta_{1}+\beta_{2}+\beta_{3}\right) P_{0}(t)+\alpha_{1} P_{1}(t)+\alpha_{2} P_{2}(t)+\alpha_{3} P_{3}(t)+\mu P_{4}(t) \\
& \frac{d P_{1}(t)}{d t}=-\left(\lambda+\alpha_{1}+\beta_{2}\right) P_{1}(t)+\beta_{1} P_{0}(t)+\alpha_{2} P_{5}(t)+\mu P_{6}(t) \\
& \frac{d P_{2}(t)}{d t}=-\left(\lambda+\alpha_{2}+\beta_{3}\right) P_{2}(t)+\beta_{2} P_{0}(t)+\alpha_{3} P_{7}(t)+\mu P_{8}(t) \\
& \frac{d P_{3}(t)}{d t}=-\left(\lambda+\alpha_{3}+\beta_{1}\right) P_{3}(t)+\beta_{3} P_{0}(t)+\alpha_{1} P_{9}(t)+\mu P_{10}(t) \\
& \frac{d P_{4}(t)}{d t}=-\mu P_{4}(t)+\lambda P_{0}(t)+\alpha_{1} P_{6}(t)+\alpha_{2} P_{8}(t) \\
& \frac{d P_{5}(t)}{d t}=-\alpha_{2} P_{5}(t)+\beta_{2} P_{1}(t) \\
& \frac{d P_{6}(t)}{d t}=-\left(\mu+\alpha_{1}\right) P_{6}(t)+\lambda P_{1}(t) \\
& \frac{d P_{7}(t)}{d t}=-\alpha_{3} P_{7}(t)+\beta_{2} P_{2}(t) \\
& \frac{d P_{8}(t)}{d t}=-\left(\mu+\alpha_{2}\right) P_{8}(t)+\lambda P_{2}(t) \\
& \frac{d P_{9}(t)}{d t}=-\alpha_{1} P_{9}(t)+\beta_{1} P_{3}(t) \\
& \frac{d P_{10}(t)}{d t}=-\mu P_{10}(t)+\lambda P_{3}(t)
\end{aligned}
$$

The differential equations above can be put in matrix form as $\dot{P}=A P$ where

$$
A=\left[\begin{array}{ccccccccccc}
-\left(\lambda+\beta_{1}+\beta_{2}+\beta_{3}\right) & \alpha_{1} & \alpha_{2} & \alpha_{3} & \mu & 0 & 0 & 0 & 0 & 0 & 0 \\
\beta_{1} & -\left(\lambda+\alpha_{1}+\beta_{2}\right) & 0 & 0 & 0 & \alpha_{2} & \mu & 0 & 0 & 0 & 0 \\
\beta_{1} & 0 & -\left(\lambda+\alpha_{2}+\beta_{3}\right) & 0 & 0 & 0 & 0 & \alpha_{3} & \mu & 0 & 0 \\
\beta_{3} & 0 & 0 & -\left(\lambda+\alpha_{3}+\beta_{1}\right) & 0 & 0 & 0 & 0 & 0 & \alpha_{1} & \mu \\
\lambda & 0 & 0 & 0 & -\mu & 0 & \alpha_{1} & 0 & \alpha_{2} & 0 & 0 \\
0 & \beta_{2} & 0 & 0 & 0 & -\alpha_{2} & 0 & 0 & 0 & 0 & 0 \\
0 & \lambda & 0 & 0 & 0 & 0 & -\left(\mu+\alpha_{1}\right) & 0 & 0 & 0 & 0 \\
0 & 0 & \beta_{3} & 0 & 0 & 0 & 0 & -\alpha_{3} & 0 & 0 & 0 \\
0 & 0 & \lambda & 0 & 0 & 0 & 0 & 0 & -\left(\mu+\alpha_{2}\right) & 0 & 0 \\
0 & 0 & 0 & \beta_{1} & 0 & 0 & 0 & 0 & 0 & -\alpha_{1} & 0 \\
0 & 0 & 0 & \lambda & 0 & 0 & 0 & 0 & 0 & 0 & -\mu
\end{array}\right]
$$

It is difficult to evaluate the transient solutions hence following El-Said[10], Haggag[11], El-Said and Shrbeny[12], and Wang et al[14], we delete the rows and columns of absorbing state of matrix $A$ and take the transpose to produce a new matrix, say $Q$.

The expected time to reach an absorbing state is obtained from

$$
E\left[T_{P(0) \rightarrow P(\text { absorbing })}\right]=P(0)\left(-Q^{-1}\right)\left[\begin{array}{l}
1 \\
1 \\
1 \\
1
\end{array}\right]
$$

Where $Q=\left[\begin{array}{cccc}-\left(\lambda+\beta_{1}+\beta_{2}+\beta_{3}\right) & \beta_{1} & \beta_{2} & \beta_{3} \\ \alpha_{1} & -\left(\lambda+\alpha_{1}+\beta_{2}\right) & 0 & 0 \\ \alpha_{2} & 0 & -\left(\lambda+\alpha_{2}+\beta_{3}\right) & 0 \\ \alpha_{3} & 0 & 0 & -\left(\lambda+\alpha_{3}+\beta_{1}\right)\end{array}\right]$

This method is successful of the following relations: 


$$
E\left[T_{P(0) \rightarrow P(\text { absorbing })}\right]=P(0) \int_{0}^{\infty} e^{A t} d t \int_{0}^{\infty} e^{A t} d t=-A^{-1}, \text { for } A^{-1}<0
$$

Expression for MTSF can therefore be obtain from

$$
E\left[T_{P(0) \rightarrow P(\text { absorbing })}\right]=M T S F=\frac{N_{1}}{D_{1}}
$$

Where

$$
\begin{gathered}
N_{1}=\left(\lambda+\alpha_{1}+\beta_{2}\right)\left(\lambda+\alpha_{2}+\beta_{3}\right)\left(\lambda+\alpha_{3}+\beta_{1}\right)+\beta_{1}\left(\lambda+\alpha_{2}+\beta_{3}\right)\left(\lambda+\alpha_{3}+\beta_{1}\right)+\beta_{1}\left(\lambda+\alpha_{1}+\beta_{2}\right)\left(\lambda+\alpha_{3}+\beta_{1}\right) \\
\beta_{3}\left(\lambda+\alpha_{1}+\beta_{2}\right)\left(\lambda+\alpha_{2}+\beta_{3}\right) \\
D_{1}=\alpha_{1} \beta_{3}^{2} \lambda+\beta_{1} \beta_{2}^{2} \lambda+\alpha_{1} \beta_{1} \beta_{3}^{2}+\alpha_{2} \beta_{1} \lambda^{2}+\alpha_{3} \beta_{2}^{2} \lambda+\alpha_{2} \beta_{2}^{2} \lambda+\beta_{1} \beta_{2} \beta_{3}^{2}+\alpha_{1} \alpha_{2} \beta_{2} \lambda+\alpha_{1} \alpha_{2} \alpha_{3} \beta_{2}+\alpha_{1} \alpha_{2} \beta_{1} \beta_{2}+ \\
\alpha_{1} \alpha_{2} \alpha_{3} \lambda+\alpha_{1} \alpha_{3} \beta_{3} \lambda+2 \alpha_{1} \beta_{1} \beta_{3} \lambda+2 \alpha_{2} \alpha_{3} \beta_{2} \lambda+2 \alpha_{2} \beta_{1} \beta_{2} \lambda+2 \alpha_{3} \beta_{2} \beta_{3} \lambda+4 \beta_{1} \beta_{2} \beta_{3} \lambda+\alpha_{3} \beta_{2} \beta_{3} \lambda-\alpha_{1} \alpha_{2} \alpha_{3} \beta_{1}+ \\
\alpha_{3} \beta_{1} \beta_{2} \lambda+\alpha_{3} \beta_{1} \beta_{2} \beta_{3}+\alpha_{1} \alpha_{3} \beta_{2} \lambda+\alpha_{1} \beta_{1} \beta_{2} \lambda+\alpha_{2} \alpha_{3} \lambda^{2}+\alpha_{1} \alpha_{2} \lambda^{2}+\lambda^{4}+\alpha_{3} \lambda^{3}+2 \beta_{1} \lambda^{3}+\alpha_{2} \lambda^{3}+2 \beta_{3} \lambda^{3}+\alpha_{1} \lambda^{3}+ \\
2 \beta_{2} \lambda^{3}+\beta_{1}^{2} \lambda^{2}+\beta_{2}^{2} \lambda^{2}+\beta_{3}^{2} \lambda^{2}+2 \alpha_{1} \beta_{3} \lambda^{2}+2 \alpha_{2} \beta_{2} \lambda^{2}+3 \beta_{2} \beta_{3} \lambda^{2}+\beta_{1}^{2} \beta_{3} \lambda-\alpha_{1} \alpha_{2} \beta_{1}^{2}+\beta_{1}^{2} \beta_{2} \lambda+\beta_{1}^{2} \beta_{2} \beta_{3}+ \\
\alpha_{1} \beta_{2} \lambda^{2}+\alpha_{2} \beta_{3} \lambda^{2}+\alpha_{3} \beta_{3} \lambda^{2}+3 \beta_{1} \beta_{3} \lambda^{2}+\alpha_{1} \alpha_{3} \lambda^{2}+\alpha_{1} \beta_{1} \lambda^{2}+2 \alpha_{3} \beta_{2} \lambda^{2}+3 \beta_{1} \beta_{2} \lambda^{2}+\alpha_{3} \beta_{1} \lambda^{2}+\alpha_{2} \alpha_{3} \beta_{2}^{2}+\alpha_{2} \beta_{1} \beta_{2}^{2} \\
+\beta_{2}^{2} \beta_{3} \lambda+\alpha_{3} \beta_{2}^{2} \beta_{3}+\beta_{1} \beta_{2}^{2} \beta_{3}+\beta_{1} \beta_{3}^{2} \lambda+\beta_{2} \beta_{3}^{2} \lambda+\alpha_{1} \beta_{2} \beta_{3} \lambda+\alpha_{1} \alpha_{3} \beta_{2} \beta_{3}+\alpha_{1} \beta_{1} \beta_{2} \beta_{3}+\alpha_{2} \beta_{1} \beta_{3} \lambda+\alpha_{1} \alpha_{2} \beta_{3} \lambda+ \\
\alpha_{1} \alpha_{2} \beta_{1} \beta_{3}+\alpha_{2} \beta_{2} \beta_{3} \lambda+\alpha_{2} \beta_{1} \beta_{2} \beta_{3}
\end{gathered}
$$

\subsection{Steady state availability Analysis for System}

For the availability case of Figure 1 following El-Said[10], Haggag[11], El-Said and Shrbeny[12], and Wang et al[14], the initial conditions for this system are:

$$
P(0)=\left[P_{0}(0), P_{1}(0), P_{2}(0), P_{3}(0), P_{4}(0), P_{5}(0), P_{6}(0), P_{7}(0), P_{8}(0), P_{9}(0), P_{10}(0)\right]=[1,0,0,0,0,0,0,0,0]
$$

The system of differential equations in for System 1 above can be expressed as:

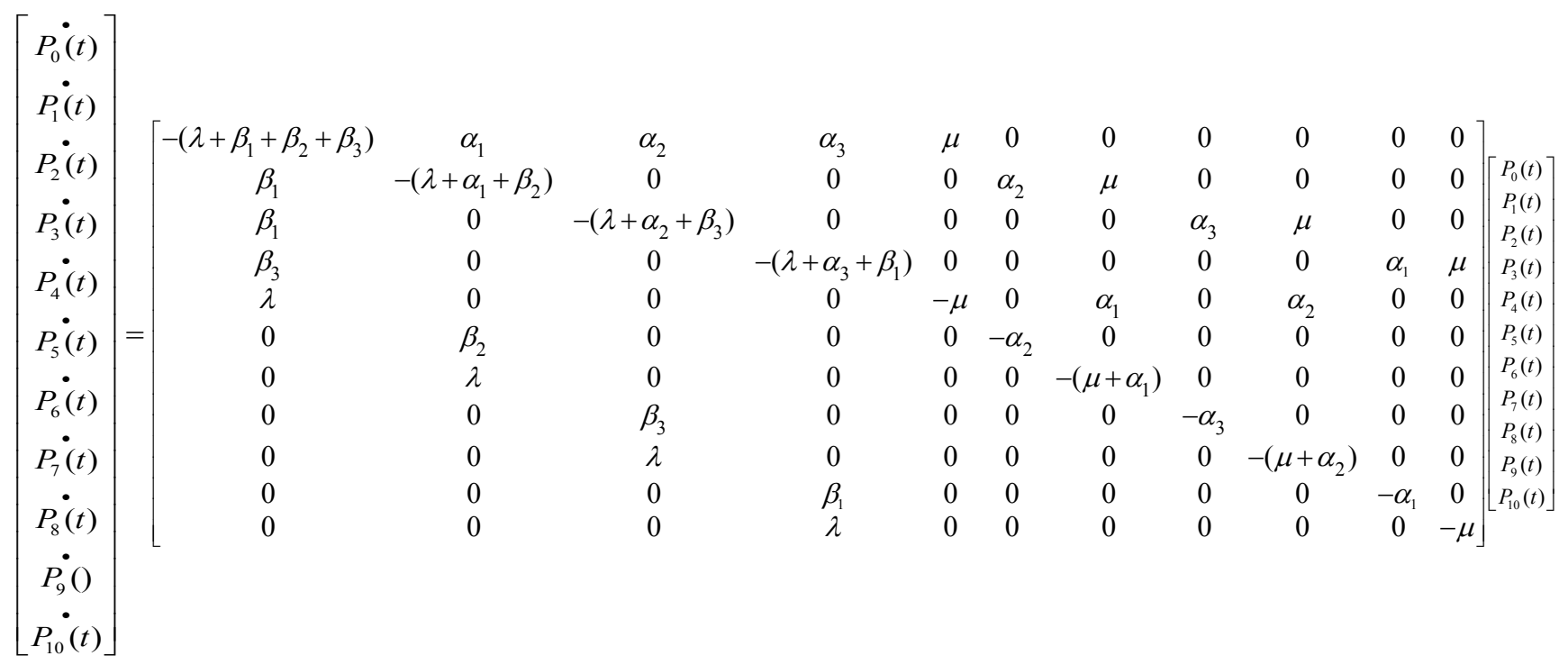

The steady-state availability is given by

$$
A_{V}(\infty)=P_{0}(\infty)+P_{1}(\infty)+P_{2}(\infty)+P_{4}(\infty)+P_{7}(\infty)
$$

In the steady state, the derivatives of the state probabilities become zero so that 
which in matrix form

$$
A P=0
$$

$\left[\begin{array}{ccccccccccc}-\left(\lambda+\beta_{1}+\beta_{2}+\beta_{3}\right) & \alpha_{1} & \alpha_{2} & \alpha_{3} & \mu & 0 & 0 & 0 & 0 & 0 & 0 \\ \beta_{1} & -\left(\lambda+\alpha_{1}+\beta_{2}\right) & 0 & 0 & 0 & \alpha_{2} & \mu & 0 & 0 & 0 & 0 \\ \beta_{1} & 0 & -\left(\lambda+\alpha_{2}+\beta_{3}\right) & 0 & 0 & 0 & 0 & \alpha_{3} & \mu & 0 & 0 \\ \beta_{3} & 0 & 0 & -\left(\lambda+\alpha_{3}+\beta_{1}\right) & 0 & 0 & 0 & 0 & 0 & \alpha_{1} & \mu \\ \lambda & 0 & 0 & 0 & -\mu & 0 & \alpha_{1} & 0 & \alpha_{2} & 0 & 0 \\ 0 & \beta_{2} & 0 & 0 & 0 & -\alpha_{2} & 0 & 0 & 0 & 0 & 0 \\ 0 & \lambda & 0 & 0 & 0 & 0 & -\left(\mu+\alpha_{1}\right) & 0 & 0 & 0 & 0 \\ 0 & 0 & \beta_{3} & 0 & 0 & 0 & 0 & -\alpha_{3} & 0 & 0 & 0 \\ 0 & 0 & \lambda & 0 & 0 & 0 & 0 & 0 & -\left(\mu+\alpha_{2}\right) & 0 & 0 \\ 0 & 0 & 0 & \beta_{1} & 0 & 0 & 0 & 0 & 0 & -\alpha_{1} & 0 \\ 0 & 0 & 0 & \lambda & 0 & 0 & 0 & 0 & 0 & 0 & -\mu\end{array}\right]\left[\begin{array}{c}P_{0}(t) \\ P_{1}(t) \\ P_{2}(t) \\ P_{3}(t) \\ P_{4}(t) \\ P_{5}(t) \\ P_{6}(t) \\ P_{7}(t) \\ P_{8}(t) \\ P_{9}(t) \\ P_{10}(t)\end{array}\right]=\left[\begin{array}{l}0 \\ 0 \\ 0 \\ 0 \\ 0 \\ 0 \\ 0 \\ 0 \\ 0 \\ 0 \\ 0\end{array}\right.$

Using the following normalizing condition

$$
P_{0}(\infty)+P_{1}(\infty)+P_{2}(\infty)+P_{3}(\infty)+P_{4}(\infty)+P_{5}(\infty)+P_{6}(\infty)+P_{7}(\infty)+P_{8}(\infty)+P_{9}(\infty)+P_{10}(\infty)=1
$$

We substitute (5) in any of the redundant rows in (4) to give

$\left[\begin{array}{ccccccccccc}-\left(\lambda+\beta_{1}+\beta_{2}+\beta_{3}\right) & \alpha_{1} & \alpha_{2} & \alpha_{3} & \mu & 0 & 0 & 0 & 0 & 0 & 0 \\ \beta_{1} & -\left(\lambda+\alpha_{1}+\beta_{2}\right) & 0 & 0 & 0 & \alpha_{2} & \mu & 0 & 0 & 0 & 0 \\ \beta_{1} & 0 & -\left(\lambda+\alpha_{2}+\beta_{3}\right) & 0 & 0 & 0 & 0 & \alpha_{3} & \mu & 0 & 0 \\ \beta_{3} & 0 & 0 & -\left(\lambda+\alpha_{3}+\beta_{1}\right) & 0 & 0 & 0 & 0 & 0 & \alpha_{1} & \mu \\ \lambda & 0 & 0 & 0 & -\mu & 0 & \alpha_{1} & 0 & \alpha_{2} & 0 & 0 \\ 0 & \beta_{2} & 0 & 0 & 0 & -\alpha_{2} & 0 & 0 & 0 & 0 & 0 \\ 0 & \lambda & 0 & 0 & 0 & 0 & -\left(\mu+\alpha_{1}\right) & 0 & 0 & 0 & 0 \\ 0 & 0 & \beta_{3} & 0 & 0 & 0 & 0 & -\alpha_{3} & 0 & 0 & 0 \\ 0 & 0 & \lambda & 0 & 0 & 0 & 0 & 0 & -\left(\mu+\alpha_{2}\right) & 0 & 0 \\ 0 & 0 & 0 & \beta_{1} & 0 & 0 & 0 & 0 & 0 & -\alpha_{1} & 0 \\ P_{1}(\infty) \\ P_{3}(\infty) \\ P_{4}(\infty) \\ P_{5}(\infty) \\ P_{6}(\infty) \\ P_{7}(\infty) \\ P_{8}(\infty) \\ P_{9}(\infty) \\ P_{10}(\infty)\end{array}\right]=\left[\begin{array}{l}0 \\ 0 \\ 0 \\ 0 \\ 1\end{array}\right.$

We solve for the system of equations in the matrix above to obtain the steady-state probabilities

$$
\begin{gathered}
P_{0}(\infty), P_{1}(\infty), P_{2}(\infty), P_{3}(\infty) \\
A_{V}=\frac{N_{2}}{D_{2}}
\end{gathered}
$$

Where

$N_{2}=\alpha_{1} \alpha_{2} \alpha_{3} \mu\left(\mu^{2}+2 \mu \lambda+\alpha_{1} \mu+\alpha_{2} \mu+\lambda^{2}+\alpha_{1} \lambda+\alpha_{2} \lambda+\alpha_{1} \alpha_{2}\right)+\alpha_{2} \alpha_{3} \beta_{1} \mu\left(\mu^{2}+\mu \lambda+\alpha_{1} \mu+\alpha_{2} \mu+\alpha_{1} \lambda+\alpha_{1} \alpha_{2}\right)+$ $\alpha_{1} \alpha_{3} \beta_{1} \mu\left(\mu^{2}+\mu \lambda+\alpha_{1} \mu+\alpha_{2} \mu+\alpha_{2} \lambda+\alpha_{1} \alpha_{2}\right)-\alpha_{1} \alpha_{2} \mu\left(-\beta_{2} \mu+\beta_{1} \mu^{2}-\beta_{3} \mu^{2}+2 \beta_{1} \mu \lambda-2 \beta_{2} \mu \lambda-2 \beta_{3} \mu \lambda-\alpha_{1} \beta_{2} \mu+\right.$ $\alpha_{1} \beta_{1} \mu-\alpha_{1} \beta_{3} \mu-\alpha_{2} \beta_{2} \mu+\alpha_{2} \beta_{1} \mu-\alpha_{2} \beta_{3} \mu-\beta_{2} \lambda^{2}+\beta_{1} \lambda^{2}-\beta_{3} \lambda^{2}-\alpha_{1} \beta_{2} \lambda+\alpha_{1} \beta_{1} \lambda-\alpha_{1} \beta_{3} \lambda-\alpha_{2} \beta_{2} \lambda+\alpha_{2} \beta_{1} \lambda-$

$$
\left.\alpha_{2} \beta_{3} \lambda-\alpha_{1} \alpha_{2} \beta_{2}+\alpha_{1} \alpha_{2} \beta_{1}-\alpha_{1} \alpha_{2} \beta_{3}\right)
$$

$D_{2}=\alpha_{1}^{2} \alpha_{2}^{2} \alpha_{3} \lambda+2 \alpha_{1} \alpha_{3} \beta_{1} \mu^{2} \lambda+\alpha_{1} \alpha_{3} \beta_{1} \mu \lambda^{2}-2 \alpha_{2} \beta_{1}^{2} \mu^{2} \lambda-\alpha_{2} \beta_{1}^{2} \mu \lambda^{2}-\alpha_{1} \alpha_{2} \beta_{1}^{2} \mu \lambda+\alpha_{1}^{2} \alpha_{3} \beta_{1} \mu \lambda+\alpha_{3} \beta_{1} \beta_{2} \mu^{2} \lambda+$

$$
\begin{aligned}
& \alpha_{1} \alpha_{3} \beta_{1} \beta_{2} \mu \lambda+\alpha_{1}^{2} \alpha_{2}^{2} \alpha_{3} \beta_{2}+\alpha_{1} \alpha_{2}^{2} \alpha_{3} \beta_{2} \lambda-\alpha_{1}^{2} \alpha_{2}^{2} \alpha_{3} \beta_{1}+\alpha_{1} \alpha_{2} \alpha_{3} \mu^{3}+\alpha_{1}^{2} \alpha_{2} \alpha_{3} \mu^{2}+\alpha_{1} \alpha_{2}^{2} \alpha_{3} \mu^{2}+\alpha_{1}^{2} \alpha_{2}^{2} \alpha_{3} \mu+ \\
& \alpha_{1} \alpha_{2} \beta_{3} \mu^{3}+\alpha_{1} \alpha_{2} \beta_{2} \mu^{3}+\alpha_{1} \alpha_{3} \beta_{1} \mu^{3}+\alpha_{1} \alpha_{2} \alpha_{3} \beta_{1} \beta_{2} \mu+\alpha_{2}^{2} \alpha_{3} \beta_{1} \mu \lambda+2 \alpha_{2} \alpha_{3} \beta_{1} \mu^{2} \lambda+3 \alpha_{1} \alpha_{2} \beta_{3} \mu^{2} \lambda+\alpha_{2}^{2} \beta_{1} \beta_{3} \mu^{2}+ \\
& \alpha_{1} \alpha_{2} \beta_{1} \beta_{2} \mu^{2}+\alpha_{1} \alpha_{2}^{2} \beta_{3} \mu^{2}+3 \alpha_{1} \alpha_{2} \beta_{2} \mu \lambda^{2}+2 \alpha_{1} \alpha_{2} \alpha_{3} \beta_{2} \mu \lambda+2 \alpha_{1}^{2} \alpha_{2} \alpha_{3} \mu \lambda+\alpha_{1}^{2} \alpha_{2}^{2} \beta_{3} \mu+2 \alpha_{1}^{2} \alpha_{2} \beta_{3} \mu \lambda+\alpha_{1}^{2} \alpha_{2} \beta_{1} \beta_{3} \mu-
\end{aligned}
$$




$$
\begin{aligned}
& 3 \alpha_{1} \alpha_{2} \beta_{1} \mu \lambda^{2}+\alpha_{2} \beta_{1} \beta_{3} \mu \lambda^{2}+\alpha_{2} \beta_{1} \beta_{3} \mu \lambda^{2}-2 \alpha_{1} \alpha_{2}^{2} \beta_{1} \mu \lambda+\alpha_{2}^{2} \beta_{1} \beta_{3} \mu \lambda+\alpha_{2}^{2} \beta_{1} \beta_{2} \mu \lambda-\alpha_{1}^{2} \alpha_{2}^{2} \beta_{1} \mu+\alpha_{1}^{2} \alpha_{2}^{2} \beta_{2} \mu+ \\
& \alpha_{1}^{2} \alpha_{2} \alpha_{3} \beta_{2} \mu-\alpha_{1} \alpha_{2}^{2} \beta_{1}^{2} \mu+\alpha_{1} \alpha_{2}^{2} \alpha_{3} \beta_{2} \mu+\alpha_{1} \alpha_{2}^{2} \beta_{1} \beta_{3} \mu+2 \alpha_{1} \alpha_{2}^{2} \alpha_{3} \mu \lambda+2 \alpha_{1} \alpha_{2} \alpha_{3} \beta_{1} \mu \lambda+2 \alpha_{1} \alpha_{2} \beta_{1} \beta_{3} \mu \lambda+ \\
& 2 \alpha_{1} \alpha_{2}^{2} \beta_{3} \mu \lambda+2 \alpha_{1} \alpha_{2}^{2} \beta_{2} \mu \lambda+\alpha_{2} \alpha_{3} \beta_{1} \mu \lambda^{2}+2 \alpha_{1}^{2} \alpha_{2} \beta_{2} \mu \lambda+\alpha_{1} \alpha_{2}^{2} \beta_{1} \beta_{2} \mu-\alpha_{1}^{2} \alpha_{2}^{2} \beta_{1} \lambda+\alpha_{1}^{2} \alpha_{2}^{2} \beta_{3} \lambda+\alpha_{1}^{2} \alpha_{2}^{2} \beta_{2} \lambda+ \\
& \alpha_{1}^{2} \alpha_{2} \alpha_{3} \beta_{2} \lambda+\alpha_{1}^{2} \alpha_{2} \alpha_{3} \lambda^{2}-\alpha_{1}^{2} \alpha_{2} \beta_{1} \lambda^{2}+\alpha_{1}^{2} \alpha_{2} \beta_{3} \lambda^{2}+\alpha_{1}^{2} \alpha_{2} \beta_{2} \lambda^{2}+\alpha_{1} \alpha_{2}^{2} \alpha_{3} \lambda^{2}-\alpha_{1} \alpha_{2}^{2} \beta_{1} \lambda^{2}+\alpha_{1} \alpha_{2}^{2} \beta_{3} \lambda^{2}+ \\
& \alpha_{1} \alpha_{2}^{2} \beta_{2} \lambda^{2}+\alpha_{1} \alpha_{2} \alpha_{3} \beta_{1} \lambda^{2}+\alpha_{1} \alpha_{2} \alpha_{3} \beta_{2} \lambda^{2}+\alpha_{1} \alpha_{2} \alpha_{3} \lambda^{3}-\alpha_{1} \alpha_{2} \beta_{1} \lambda^{3}+\alpha_{1} \alpha_{2} \beta_{3} \lambda^{3}+\alpha_{1} \alpha_{2} \beta_{2} \lambda^{3}+\alpha_{1} \alpha_{3} \beta_{1} \beta_{2} \mu^{2}+ \\
& \alpha_{1} \alpha_{2} \alpha_{3} \beta_{1} \mu^{2}+\alpha_{1} \beta_{1} \beta_{3} \mu^{2} \lambda+\alpha_{1}^{2} \alpha_{3} \beta_{1} \mu^{2}+\alpha_{2}^{2} \beta_{1} \beta_{2} \mu^{2}+\alpha_{1}^{2} \alpha_{3} \beta_{1} \beta_{2} \mu^{2}-3 \alpha_{1} \alpha_{2} \beta_{1} \mu^{2} \lambda+2 \alpha_{2} \beta_{1} \beta_{3} \mu^{2} \lambda+2 \alpha_{2} \beta_{1} \beta_{2} \mu^{2} \lambda+ \\
& \alpha_{1}^{2} \alpha_{2} \beta_{2} \mu^{2}-\alpha_{1} \alpha_{2}^{2} \beta_{1} \mu^{2}-\alpha_{1} \alpha_{2} \beta_{1}^{2} \mu^{2}+\alpha_{2}^{2} \alpha_{3} \beta_{1} \mu^{2}-\alpha_{2}^{2} \beta_{1}^{2} \mu^{2}+\alpha_{1}^{2} \alpha_{2} \beta_{3} \mu^{2}+3 \alpha_{1} \alpha_{2} \beta_{2} \mu^{2} \lambda-\alpha_{1}^{2} \alpha_{2} \beta_{1} \mu^{2}+ \\
& \alpha_{1} \alpha_{2}^{2} \beta_{2} \mu^{2}+\alpha_{1}^{2} \beta_{1} \beta_{3} \mu^{2}+\alpha_{1} \alpha_{2} \beta_{1} \beta_{2} \mu \lambda-\alpha_{2}^{2} \beta_{1}^{2} \mu \lambda-2 \alpha_{1}^{2} \alpha_{2} \beta_{1} \mu \lambda+3 \alpha_{1} \alpha_{2} \beta_{3} \mu \lambda^{2}+3 \alpha_{1} \alpha_{2} \alpha_{3} \mu \lambda^{2}+\alpha_{1} \beta_{1} \beta_{3} \mu^{3}+ \\
& \alpha_{2} \alpha_{3} \beta_{1} \mu^{3}-\alpha_{2} \beta_{1}^{2} \mu^{3}+\alpha_{2} \beta_{1} \beta_{3} \mu^{3}+\alpha_{2} \beta_{1} \beta_{2} \mu^{3}+\alpha_{3} \beta_{1} \beta_{2} \mu^{3}+2 \alpha_{1} \alpha_{2} \beta_{1} \beta_{3} \mu^{2}+\alpha_{1} \alpha_{2} \alpha_{3} \beta_{2} \mu^{2}+3 \alpha_{1} \alpha_{2} \alpha_{3} \mu^{2} \lambda-\alpha_{1} \alpha_{2} \beta_{1} \mu^{3}
\end{aligned}
$$

\subsection{Busy Period Analysis}

Using the same in itial conditions as for the reliability case:

$$
\begin{aligned}
P(0) & =\left[P_{0}(0), P_{1}(0), P_{2}(0), P_{3}(0), P_{4}(0), P_{5}(0), P_{6}(0), P_{7}(0), P_{8}(0), P_{9}(0), P_{10}(0)\right] \\
& =[1,0,0,0,0,0,0,0,0,0,0]
\end{aligned}
$$

The differential equations can be expressed as

$$
\left[\begin{array}{c}
\dot{P_{0}}(t) \\
\dot{P_{1}}(t) \\
\dot{P_{2}}(t) \\
\dot{P_{3}(t)} \\
\dot{P_{4}(t)} \\
\dot{P_{5}(t)} \\
\dot{P_{6}}(t) \\
\dot{P_{7}}(t) \\
\dot{P_{8}(t)} \\
\dot{P_{9}} \\
\dot{P_{10}}(t)
\end{array}\right]=
$$

$\left[\begin{array}{ccccccccccc}-\left(\lambda+\beta_{1}+\beta_{2}+\beta_{3}\right) & \alpha_{1} & \alpha_{2} & \alpha_{3} & \mu & 0 & 0 & 0 & 0 & 0 & 0 \\ \beta_{1} & -\left(\lambda+\alpha_{1}+\beta_{2}\right) & 0 & 0 & 0 & \alpha_{2} & \mu & 0 & 0 & 0 & 0 \\ \beta_{1} & 0 & -\left(\lambda+\alpha_{2}+\beta_{3}\right) & 0 & 0 & 0 & 0 & \alpha_{3} & \mu & 0 & 0 \\ \beta_{3} & 0 & 0 & -\left(\lambda+\alpha_{3}+\beta_{1}\right) & 0 & 0 & 0 & 0 & 0 & \alpha_{1} & \mu \\ \lambda & 0 & 0 & 0 & -\mu & 0 & \alpha_{1} & 0 & \alpha_{2} & 0 & 0 \\ 0 & \beta_{2} & 0 & 0 & 0 & -\alpha_{2} & 0 & 0 & 0 & 0 & 0 \\ 0 & \lambda & 0 & 0 & 0 & 0 & -\left(\mu+\alpha_{1}\right) & 0 & 0 & 0 & 0 \\ 0 & 0 & \beta_{3} & 0 & 0 & 0 & 0 & -\alpha_{3} & 0 & 0 & 0 \\ 0 & 0 & \lambda & 0 & 0 & 0 & 0 & 0 & -\left(\mu+\alpha_{2}\right) & 0 & 0 \\ 0 & 0 & 0 & \beta_{1} & 0 & 0 & 0 & 0 & 0 & -\alpha_{1} & 0 \\ 0 & 0 & 0 & \lambda & 0 & 0 & 0 & 0 & 0 & 0 & -\mu\end{array}\right]\left[\begin{array}{l}P_{0}(t) \\ P_{1}(t) \\ P_{2}(t) \\ P_{3}(t) \\ P_{4}(t) \\ P_{5}(t) \\ P_{6}(t) \\ P_{7}(t) \\ P_{8}(t) \\ P_{9}(t) \\ P_{10}(t)\end{array}\right]$


In the steady state, the derivatives of the state probabilities become zero this will enable us to compute steady state busy :

\begin{tabular}{|c|c|c|c|c|c|c|c|c|c|c|c|c|}
\hline & & & $B(\infty)=1$ & $-P_{0}$ & & & & & & & & (6) \\
\hline$A P=0$ & & & & & & & & & & & & \\
\hline$-\left(\lambda+\beta_{1}+\beta_{2}+\beta_{3}\right)$ & $\alpha_{1}$ & $\alpha_{2}$ & $\alpha_{3}$ & $\mu$ & 0 & 0 & 0 & 0 & 0 & 0 & & \\
\hline$\beta_{1}$ & $-\left(\lambda+\alpha_{1}+\beta_{2}\right)$ & 0 & 0 & 0 & $\alpha_{2}$ & $\mu$ & 0 & 0 & 0 & 0 & {$\left[\begin{array}{l}P_{0}(t) \\
P(t)\end{array}\right]$} & {$\left[\begin{array}{l}0 \\
0\end{array}\right]$} \\
\hline$\beta_{1}$ & 0 & $-\left(\lambda+\alpha_{2}+\beta_{3}\right)$ & 0 & 0 & 0 & 0 & $\alpha_{3}$ & $\mu$ & 0 & 0 & $\mid \begin{array}{l}P_{1}(t) \\
P_{2}(t)\end{array}$ & $\begin{array}{l}0 \\
0\end{array}$ \\
\hline$\beta_{3}$ & 0 & 0 & $-\left(\lambda+\alpha_{3}+\beta_{1}\right)$ & 0 & 0 & 0 & 0 & 0 & $\alpha_{1}$ & $\mu$ & $P_{3}(t)$ & 0 \\
\hline$\lambda$ & 0 & 0 & 0 & $-\mu$ & 0 & $\alpha_{1}$ & 0 & $\alpha_{2}$ & 0 & 0 & $P_{4}(t)$ & 0 \\
\hline 0 & $\beta_{2}$ & 0 & 0 & 0 & $-\alpha_{2}$ & 0 & 0 & 0 & 0 & 0 & $P_{5}(t)$ & 0 \\
\hline 0 & $\lambda$ & 0 & 0 & 0 & 0 & $-\left(\mu+\alpha_{1}\right)$ & 0 & 0 & 0 & 0 & $\left|\begin{array}{l}P_{6}(t) \\
P(t)\end{array}\right|$ & $\begin{array}{l}0 \\
0\end{array}$ \\
\hline 0 & 0 & $\beta_{3}$ & 0 & 0 & 0 & 0 & $-\alpha_{3}$ & 0 & 0 & 0 & $\left|\begin{array}{l}P_{7}(t) \\
P_{8}(t)\end{array}\right|$ & $\begin{array}{l}0 \\
0\end{array} \mid$ \\
\hline 0 & 0 & $\lambda$ & 0 & 0 & 0 & 0 & 0 & $-\left(\mu+\alpha_{2}\right)$ & 0 & 0 & $\begin{array}{l}8(t) \\
P_{9}(t)\end{array}$ & 0 \\
\hline 0 & 0 & 0 & $\beta_{1}$ & 0 & 0 & 0 & 0 & 0 & $-\alpha_{1}$ & 0 & {$\left[P_{10}(t)\right]$} & 0 \\
\hline 0 & 0 & 0 & $\lambda$ & 0 & 0 & 0 & 0 & 0 & 0 & $-\mu\rfloor$ & & \\
\hline
\end{tabular}

We solve for $P_{0}(\infty)$

Using the following normalizing condition

$$
P_{0}(\infty)+P_{1}(\infty)+P_{2}(\infty)+P_{3}(\infty)+P_{4}(\infty)+P_{5}(\infty)+P_{6}(\infty)+P_{7}(\infty)+P_{8}(\infty)+P_{9}(\infty)+P_{10}(\infty)=1
$$

We substitute (5) in any of the redundant rows in (4) to give

\begin{tabular}{|c|c|c|c|c|c|c|c|c|c|c|c|}
\hline$\left[-\left(\lambda+\beta_{1}+\beta_{2}+\beta_{3}\right)\right.$ & $\alpha_{1}$ & $\alpha_{2}$ & $\alpha_{3}$ & $\mu$ & 0 & 0 & 0 & 0 & 0 & $0]$ & \\
\hline$\beta_{1}$ & $-\left(\lambda+\alpha_{1}+\beta_{2}\right)$ & 0 & 0 & 0 & $\alpha_{2}$ & $\mu$ & 0 & 0 & 0 & 0 & $\left.\begin{array}{l}P_{0}(\infty) \\
P_{1}(\infty)\end{array}\right]$ \\
\hline$\beta_{1}$ & 0 & $-\left(\lambda+\alpha_{2}+\beta_{3}\right)$ & 0 & 0 & 0 & 0 & $\alpha_{3}$ & $\mu$ & 0 & 0 & $\begin{array}{l}P_{1}(\infty) \\
P_{2}(\infty)\end{array}$ \\
\hline$\beta_{3}$ & 0 & 0 & $-\left(\lambda+\alpha_{3}+\beta_{1}\right)$ & 0 & 0 & 0 & 0 & 0 & $\alpha_{1}$ & $\mu$ & $P_{3}(\infty)$ \\
\hline$\lambda$ & 0 & 0 & 0 & $-\mu$ & 0 & $\alpha_{1}$ & 0 & $\alpha_{2}$ & 0 & 0 & $P_{4}(\infty)$ \\
\hline 0 & $\beta_{2}$ & 0 & 0 & 0 & $-\alpha_{2}$ & 0 & 0 & 0 & 0 & 0 & $P_{5}(\infty)$ \\
\hline 0 & $\lambda$ & 0 & 0 & 0 & 0 & $-\left(\mu+\alpha_{1}\right)$ & 0 & 0 & 0 & 0 & $P_{6}(\infty)$ \\
\hline 0 & 0 & $\beta_{3}$ & 0 & 0 & 0 & 0 & $-\alpha_{3}$ & 0 & 0 & 0 & $\begin{array}{l}P_{7}(\infty) \\
P_{8}(\infty)\end{array}$ \\
\hline 0 & 0 & $\lambda$ & 0 & 0 & 0 & 0 & 0 & $-\left(\mu+\alpha_{2}\right)$ & 0 & 0 & $P_{9}(\infty)$ \\
\hline 0 & 0 & 0 & $\beta_{1}$ & 0 & 0 & 0 & 0 & 0 & $-\alpha_{1}$ & 0 & $\left.P_{10}(\infty)\right]$ \\
\hline 1 & 1 & 1 & 1 & 1 & 1 & 1 & 1 & 1 & 1 & $1]$ & \\
\hline
\end{tabular}

The steady state busy period $B(\infty)$ is therefore

$$
B(\infty)=\frac{N_{3}}{D_{2}}
$$

$N_{3}=\alpha_{1} \alpha_{2} \beta_{3} \mu^{3}+\alpha_{1} \alpha_{2} \beta_{2} \mu^{3}+\alpha_{1} \alpha_{3} \beta_{1} \mu^{3}+\alpha_{1} \alpha_{2} \alpha_{3} \beta_{1} \beta_{2} \mu+\alpha_{2}^{2} \alpha_{3} \beta_{1} \mu \lambda+2 \alpha_{2} \alpha_{3} \beta_{1} \mu^{2} \lambda+3 \alpha_{1} \alpha_{2} \beta_{3} \mu^{2} \lambda+\alpha_{2}^{2} \beta_{1} \beta_{3} \mu^{2}+$ $\alpha_{1} \alpha_{2} \beta_{1} \beta_{2} \mu^{2}+\alpha_{1} \alpha_{2}^{2} \beta_{3} \mu^{2}+3 \alpha_{1} \alpha_{2} \beta_{2} \mu \lambda^{2}+2 \alpha_{1} \alpha_{2} \alpha_{3} \beta_{2} \mu \lambda+\alpha_{1}^{2} \alpha_{2} \alpha_{3} \mu \lambda+\alpha_{1}^{2} \alpha_{2}^{2} \beta_{3} \mu+2 \alpha_{1}^{2} \alpha_{2} \beta_{3} \mu \lambda+\alpha_{1}^{2} \alpha_{2} \beta_{1} \beta_{3} \mu-$ $3 \alpha_{1} \alpha_{2} \beta_{1} \mu \lambda^{2}+\alpha_{2} \beta_{1} \beta_{3} \mu \lambda^{2}+\alpha_{2} \beta_{1} \beta_{3} \mu \lambda^{2}-2 \alpha_{1} \alpha_{2}^{2} \beta_{1} \mu \lambda+\alpha_{2}^{2} \beta_{1} \beta_{3} \mu \lambda+\alpha_{2}^{2} \beta_{1} \beta_{2} \mu \lambda-\alpha_{1}^{2} \alpha_{2}^{2} \beta_{1} \mu+\alpha_{1}^{2} \alpha_{2}^{2} \beta_{2} \mu+$ $\alpha_{1}^{2} \alpha_{2} \alpha_{3} \beta_{2} \mu-\alpha_{1} \alpha_{2}^{2} \beta_{1}^{2} \mu+\alpha_{1} \alpha_{2}^{2} \alpha_{3} \beta_{2} \mu+\alpha_{1} \alpha_{2}^{2} \beta_{1} \beta_{3} \mu+\alpha_{1} \alpha_{2}^{2} \alpha_{3} \mu \lambda+2 \alpha_{1} \alpha_{2} \alpha_{3} \beta_{1} \mu \lambda+2 \alpha_{1} \alpha_{2} \beta_{1} \beta_{3} \mu \lambda+$ $2 \alpha_{1} \alpha_{2}^{2} \beta_{3} \mu \lambda+2 \alpha_{1} \alpha_{2}^{2} \beta_{2} \mu \lambda+\alpha_{2} \alpha_{3} \beta_{1} \mu \lambda^{2}+2 \alpha_{1}^{2} \alpha_{2} \beta_{2} \mu \lambda+\alpha_{1} \alpha_{2}^{2} \beta_{1} \beta_{2} \mu-\alpha_{1}^{2} \alpha_{2}^{2} \beta_{1} \lambda+\alpha_{1}^{2} \alpha_{2}^{2} \beta_{3} \lambda+\alpha_{1}^{2} \alpha_{2}^{2} \beta_{2} \lambda+$ $\alpha_{1}^{2} \alpha_{2} \alpha_{3} \beta_{2} \lambda+\alpha_{1}^{2} \alpha_{2} \alpha_{3} \lambda^{2}-\alpha_{1}^{2} \alpha_{2} \beta_{1} \lambda^{2}+\alpha_{1}^{2} \alpha_{2} \beta_{3} \lambda^{2}+\alpha_{1}^{2} \alpha_{2} \beta_{2} \lambda^{2}+\alpha_{1} \alpha_{2}^{2} \alpha_{3} \lambda^{2}-\alpha_{1} \alpha_{2}^{2} \beta_{1} \lambda^{2}+\alpha_{1} \alpha_{2}^{2} \beta_{3} \lambda^{2}+$ $\alpha_{1} \alpha_{2}^{2} \beta_{2} \lambda^{2}+\alpha_{1} \alpha_{2} \alpha_{3} \beta_{1} \lambda^{2}+\alpha_{1} \alpha_{2} \alpha_{3} \beta_{2} \lambda^{2}+\alpha_{1} \alpha_{2} \alpha_{3} \lambda^{3}-\alpha_{1} \alpha_{2} \beta_{1} \lambda^{3}+\alpha_{1} \alpha_{2} \beta_{3} \lambda^{3}+\alpha_{1} \alpha_{2} \beta_{2} \lambda^{3}+\alpha_{1} \alpha_{3} \beta_{1} \beta_{2} \mu^{2}+$ $\alpha_{1} \alpha_{2} \alpha_{3} \beta_{1} \mu^{2}+\alpha_{1} \beta_{1} \beta_{3} \mu^{2} \lambda+\alpha_{1}^{2} \alpha_{3} \beta_{1} \mu^{2}+\alpha_{2}^{2} \beta_{1} \beta_{2} \mu^{2}+\alpha_{1}^{2} \alpha_{3} \beta_{1} \beta_{2} \mu^{2}-3 \alpha_{1} \alpha_{2} \beta_{1} \mu^{2} \lambda+2 \alpha_{2} \beta_{1} \beta_{3} \mu^{2} \lambda+2 \alpha_{2} \beta_{1} \beta_{2} \mu^{2} \lambda+$ 


$$
\begin{gathered}
\alpha_{1}^{2} \alpha_{2} \beta_{2} \mu^{2}-\alpha_{1} \alpha_{2}^{2} \beta_{1} \mu^{2}-\alpha_{1} \alpha_{2} \beta_{1}^{2} \mu^{2}+\alpha_{2}^{2} \alpha_{3} \beta_{1} \mu^{2}-\alpha_{2}^{2} \beta_{1}^{2} \mu^{2}+\alpha_{1}^{2} \alpha_{2} \beta_{3} \mu^{2}+3 \alpha_{1} \alpha_{2} \beta_{2} \mu^{2} \lambda-\alpha_{1}^{2} \alpha_{2} \beta_{1} \mu^{2}+ \\
\alpha_{1} \alpha_{2}^{2} \beta_{2} \mu^{2}+\alpha_{1}^{2} \beta_{1} \beta_{3} \mu^{2}+\alpha_{1} \alpha_{2} \beta_{1} \beta_{2} \mu \lambda-\alpha_{2}^{2} \beta_{1}^{2} \mu \lambda-2 \alpha_{1}^{2} \alpha_{2} \beta_{1} \mu \lambda+3 \alpha_{1} \alpha_{2} \beta_{3} \mu \lambda^{2}+2 \alpha_{1} \alpha_{2} \alpha_{3} \mu \lambda^{2}+\alpha_{1} \beta_{1} \beta_{3} \mu^{3}+ \\
\alpha_{2} \alpha_{3} \beta_{1} \mu^{3}-\alpha_{2} \beta_{1}^{2} \mu^{3}+\alpha_{2} \beta_{1} \beta_{3} \mu^{3}+\alpha_{2} \beta_{1} \beta_{2} \mu^{3}+\alpha_{3} \beta_{1} \beta_{2} \mu^{3}+2 \alpha_{1} \alpha_{2} \beta_{1} \beta_{3} \mu^{2}+\alpha_{1} \alpha_{2} \alpha_{3} \beta_{2} \mu^{2}+\alpha_{1} \alpha_{2} \alpha_{3} \mu^{2} \lambda-\alpha_{1} \alpha_{2} \beta_{1} \mu^{3}+ \\
\alpha_{1}^{2} \alpha_{2}^{2} \alpha_{3} \lambda+2 \alpha_{1} \alpha_{3} \beta_{1} \mu^{2} \lambda+\alpha_{1} \alpha_{3} \beta_{1} \mu \lambda^{2}-2 \alpha_{2} \beta_{1}^{2} \mu^{2} \lambda-\alpha_{2} \beta_{1}^{2} \mu \lambda^{2}-\alpha_{1} \alpha_{2} \beta_{1}^{2} \mu \lambda+\alpha_{1}^{2} \alpha_{3} \beta_{1} \mu \lambda+\alpha_{3} \beta_{1} \beta_{2} \mu^{2} \lambda+ \\
\alpha_{1} \alpha_{3} \beta_{1} \beta_{2} \mu \lambda+\alpha_{1}^{2} \alpha_{2}^{2} \alpha_{3} \beta_{2}+\alpha_{1} \alpha_{2}^{2} \alpha_{3} \beta_{2} \lambda-\alpha_{1}^{2} \alpha_{2}^{2} \alpha_{3} \beta_{1}
\end{gathered}
$$

\subsection{Profit Analys is}

Following El-Said[10], Haggag[11], El-said and sherbeny[13] and Wang et al[14], the expected profit per unit time incurred to the system in the steady-state is given by:

Profit =total revenue generated from system using - total cost due to repair of failed unit or subsystem B

$$
P F=C_{0} A_{V 2}(\infty)-C_{1} B_{2}(\infty)
$$

Where $P F:$ is the profit incurred to the system

$C_{0}$ : is the revenue per unit up time of the system

$C_{1}:$ is the cost per unit time which the system is under repair

\section{Results}

The following particular cases are considered:

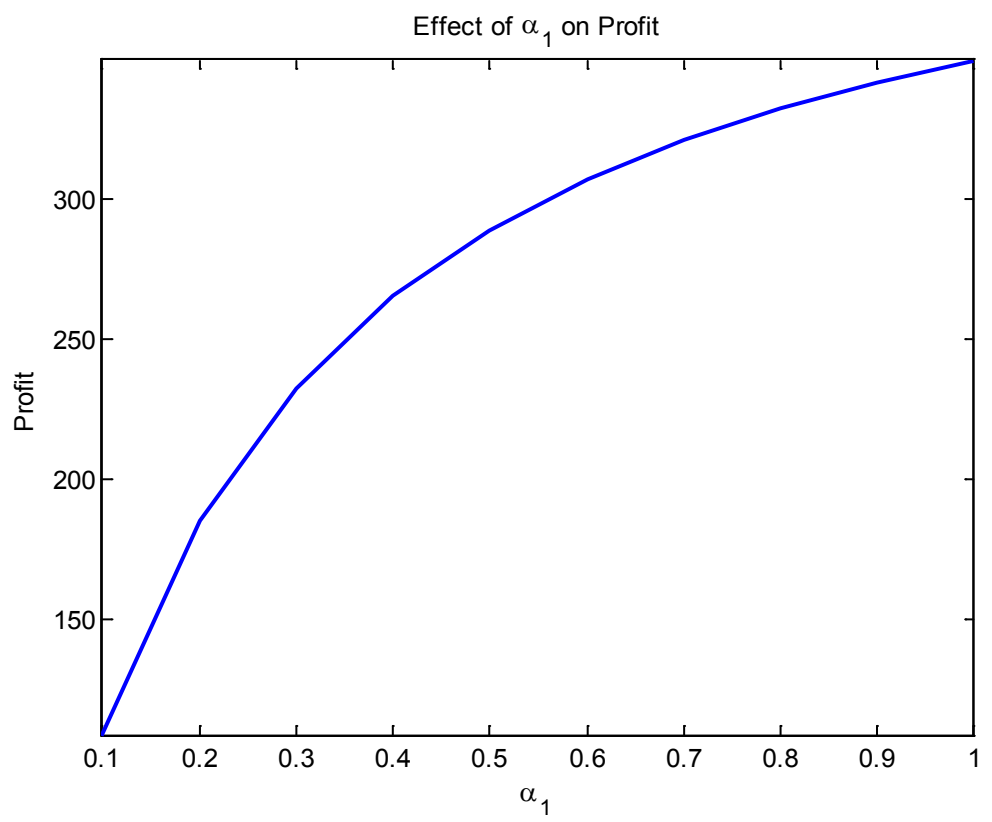

Figure 2. effect of $\alpha_{1}$ on Profit 

and Three Small Dissimilar Units

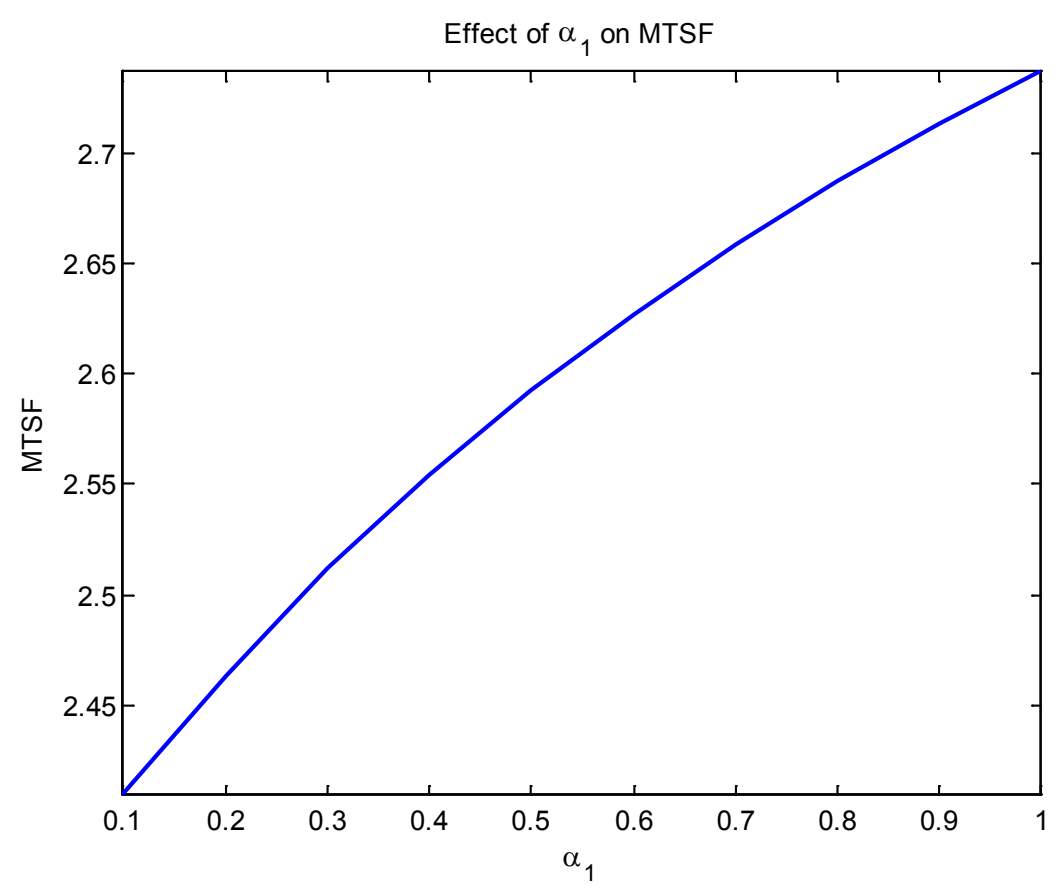

Figure 3. effect of $\alpha_{1}$ on MT SF

Effect of $\alpha_{1}$ on Avalability

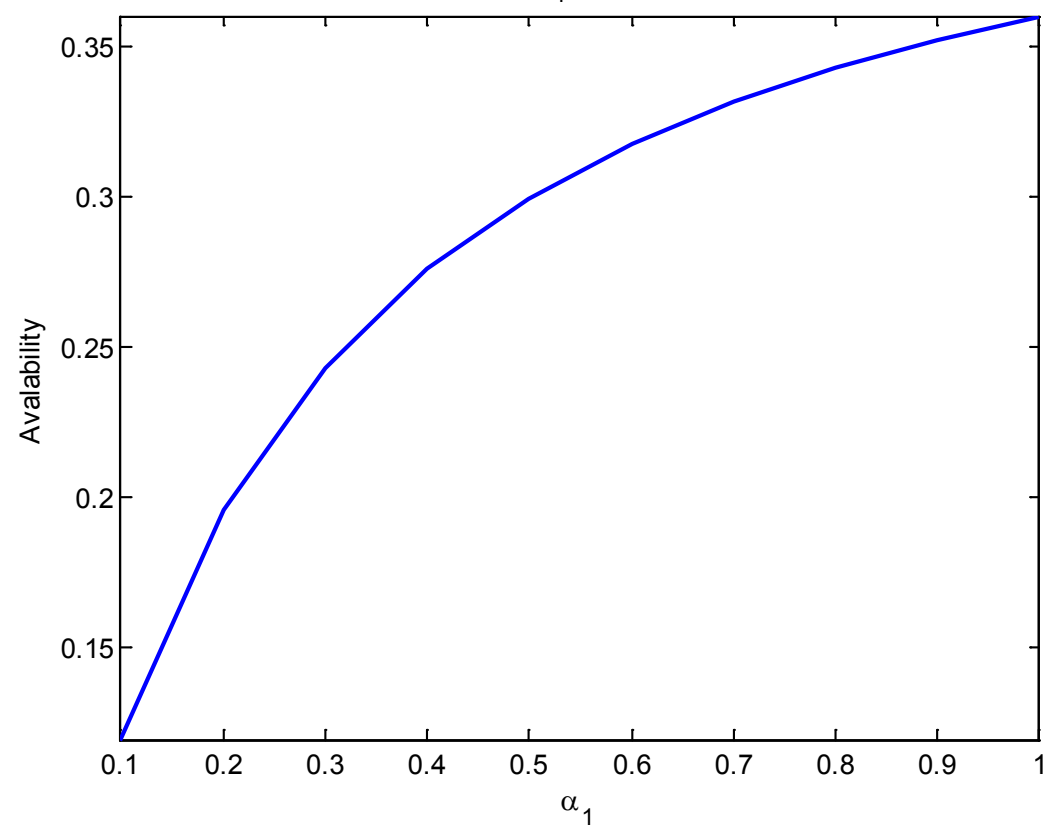

Figure 4. effect of $\alpha_{1}$ on system availability 


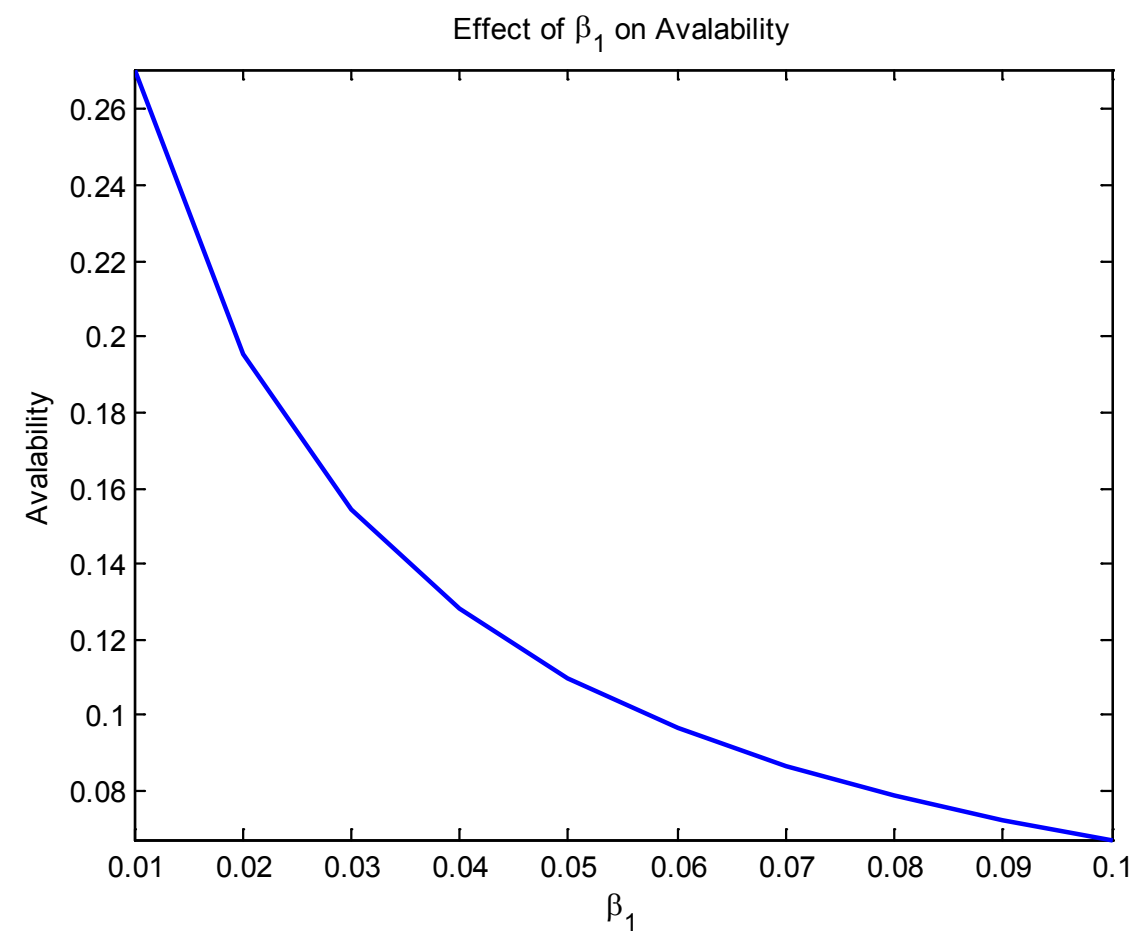

Figure 5. effect of $\beta_{1}$ on system availability

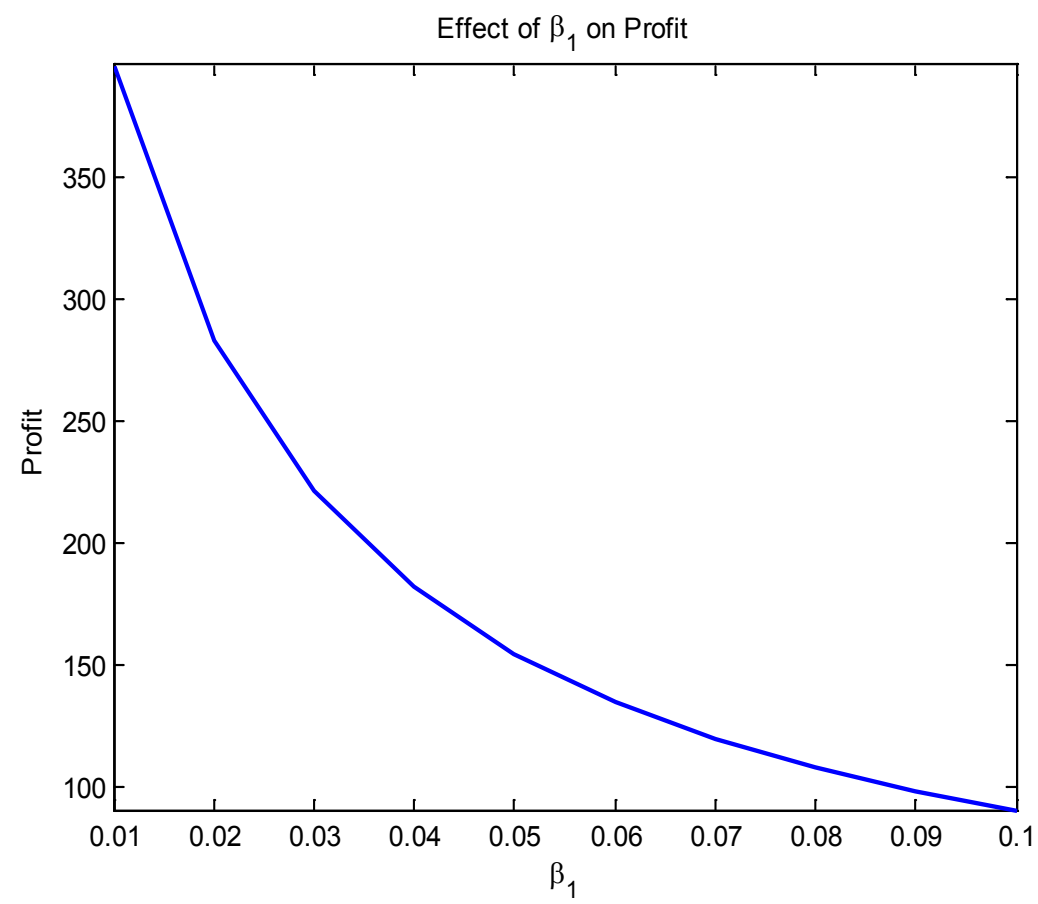

Figure 6. effect of $\beta_{1}$ on Profit 

and Three Small Dissimilar Units

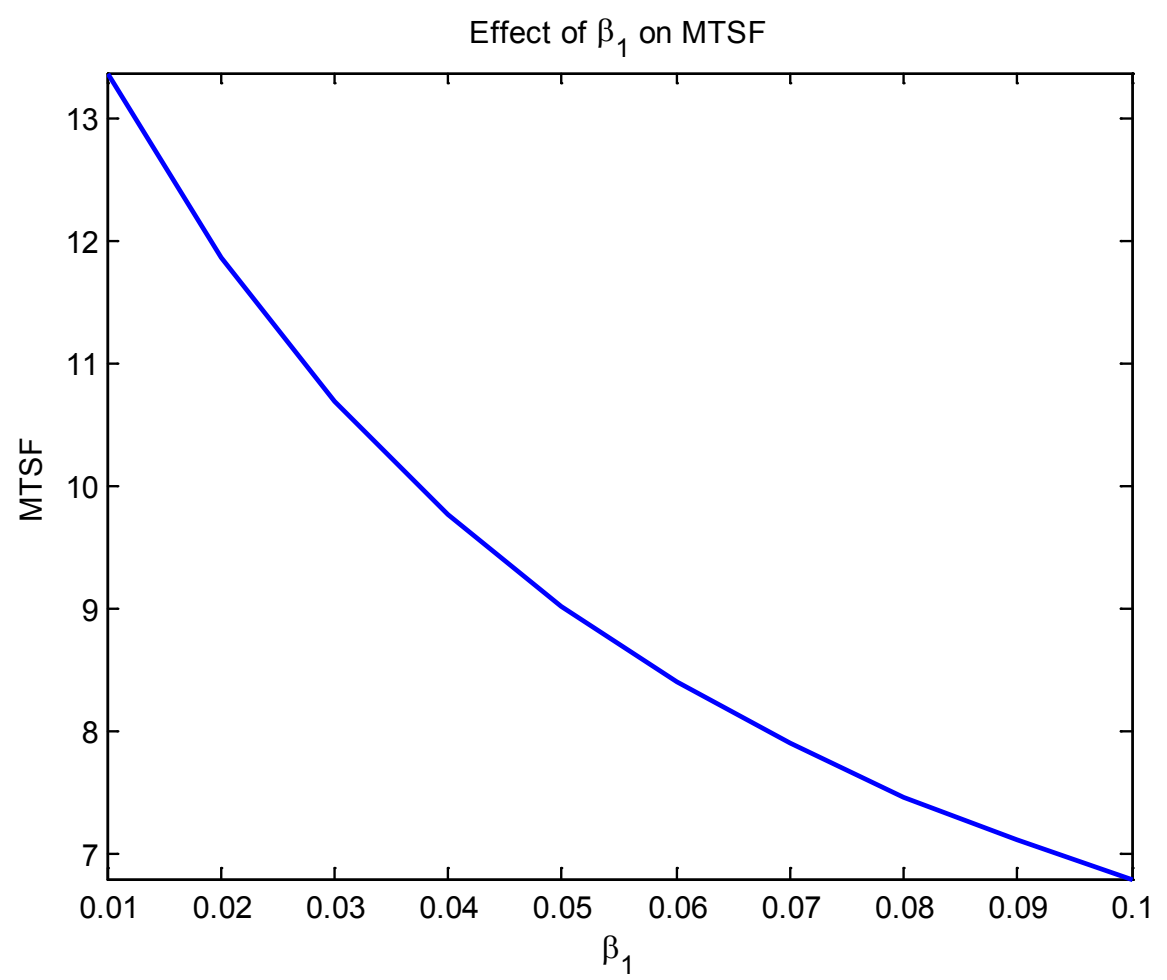

Figure 7. effect of $\beta_{1}$ on MTSF

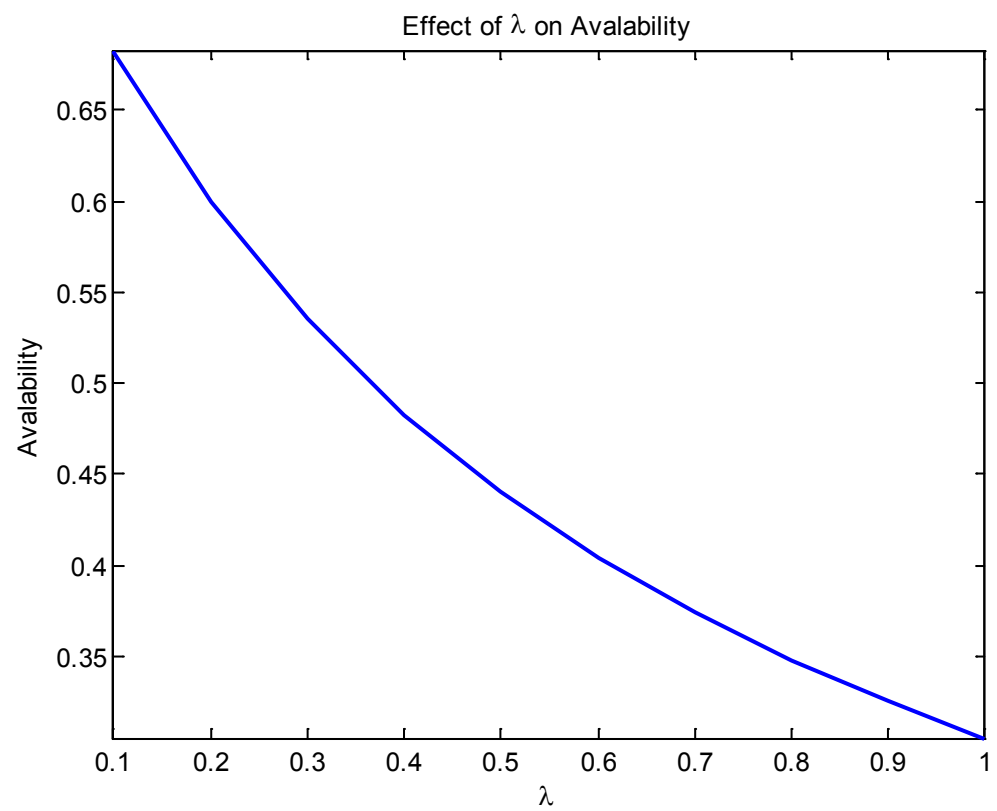

Figure 8. effect of $\lambda$ on system availability 


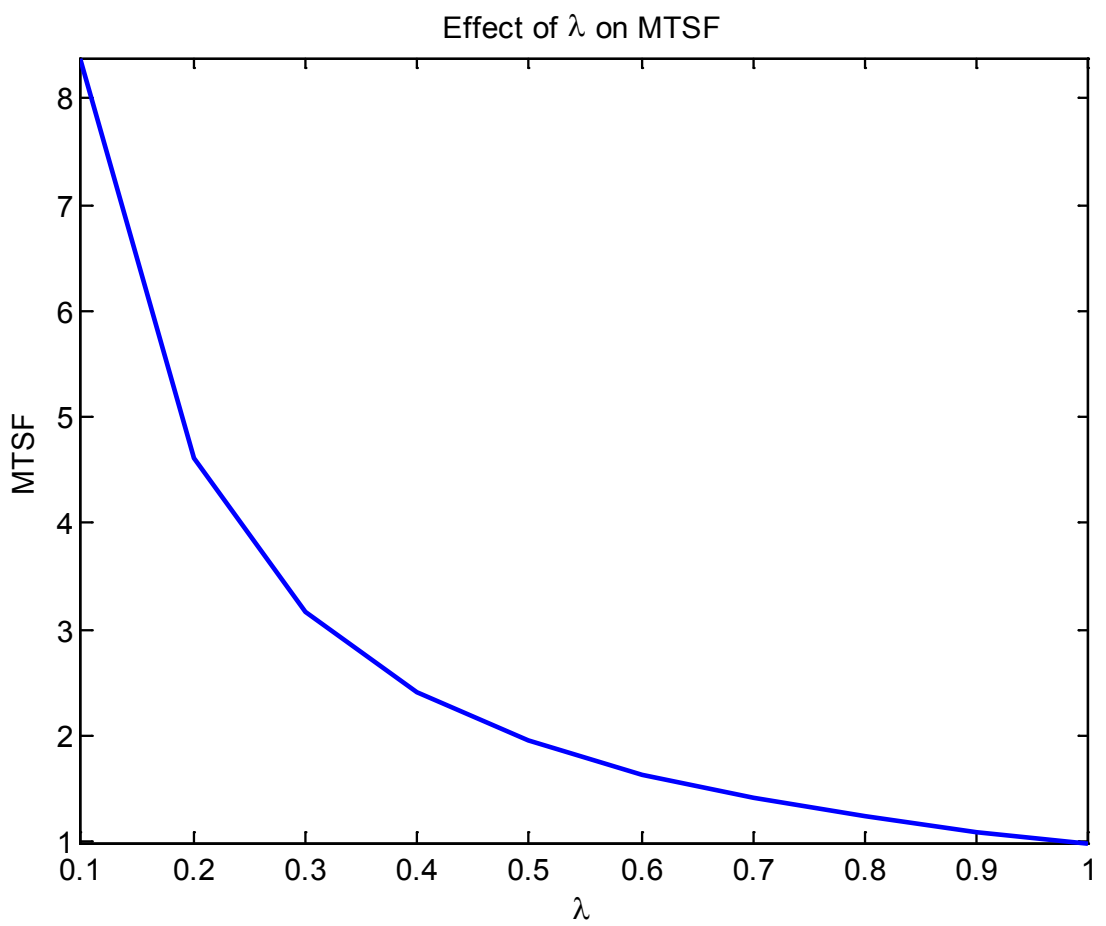

Figure 9. effect of $\lambda$ on MT SF

Effect of $\lambda$ on Profit

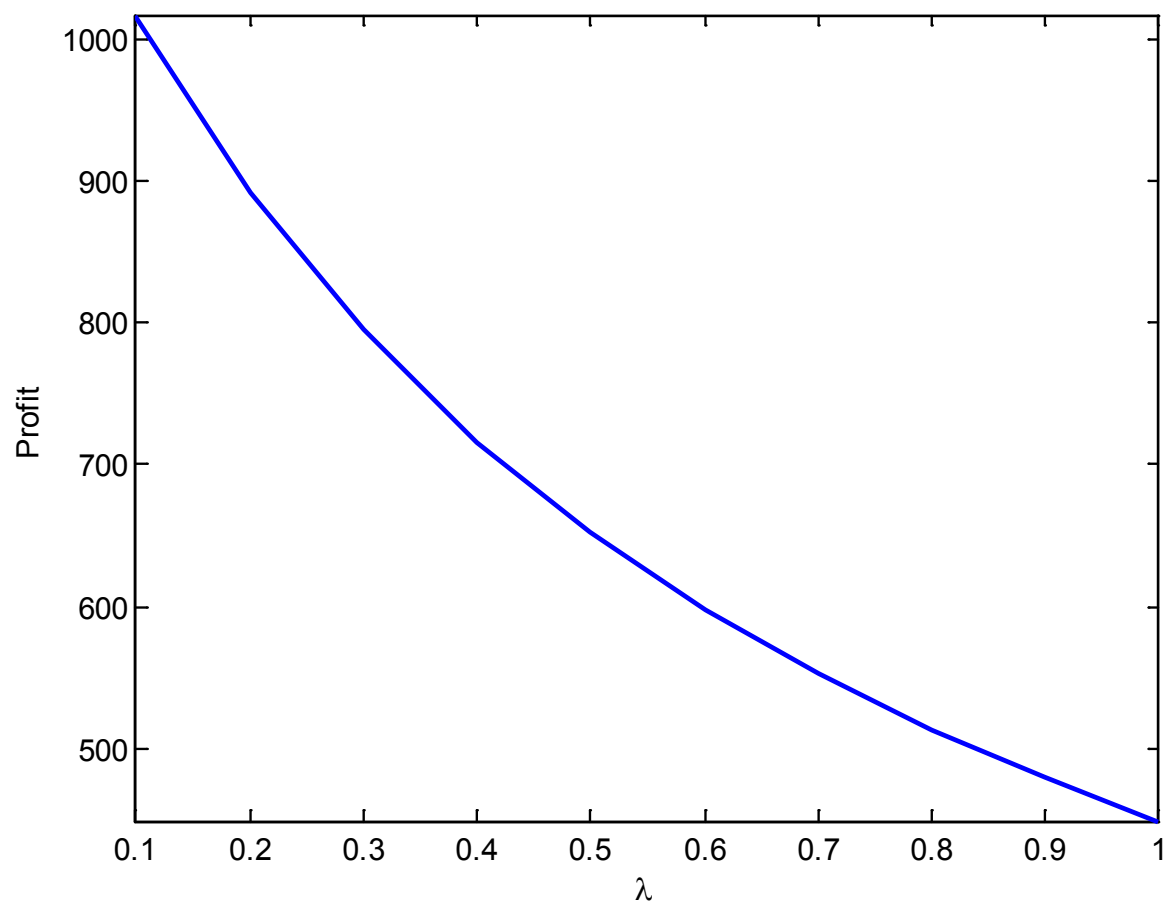

Figure 10. effect of $\lambda$ on Profit 

and Three Small Dissimilar Units

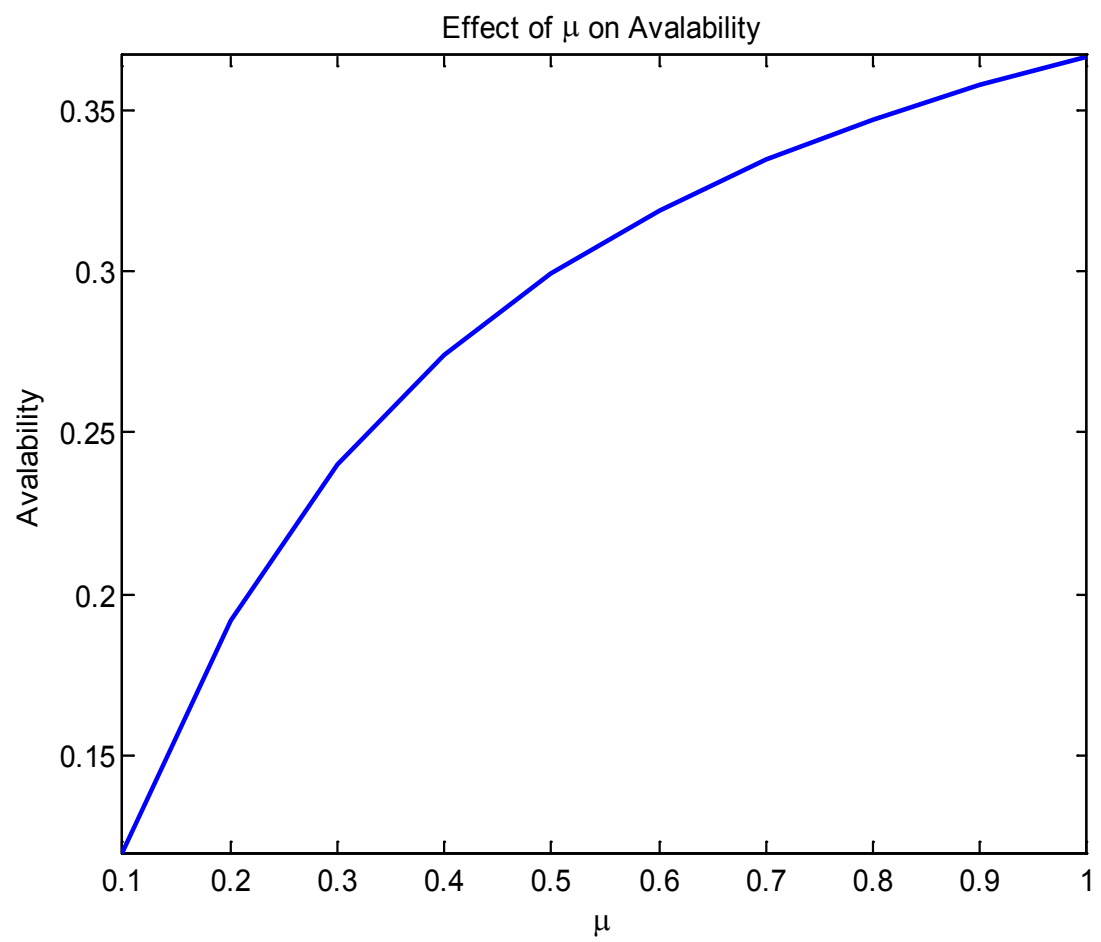

Figure 11. effect of $\mu$ on system availability

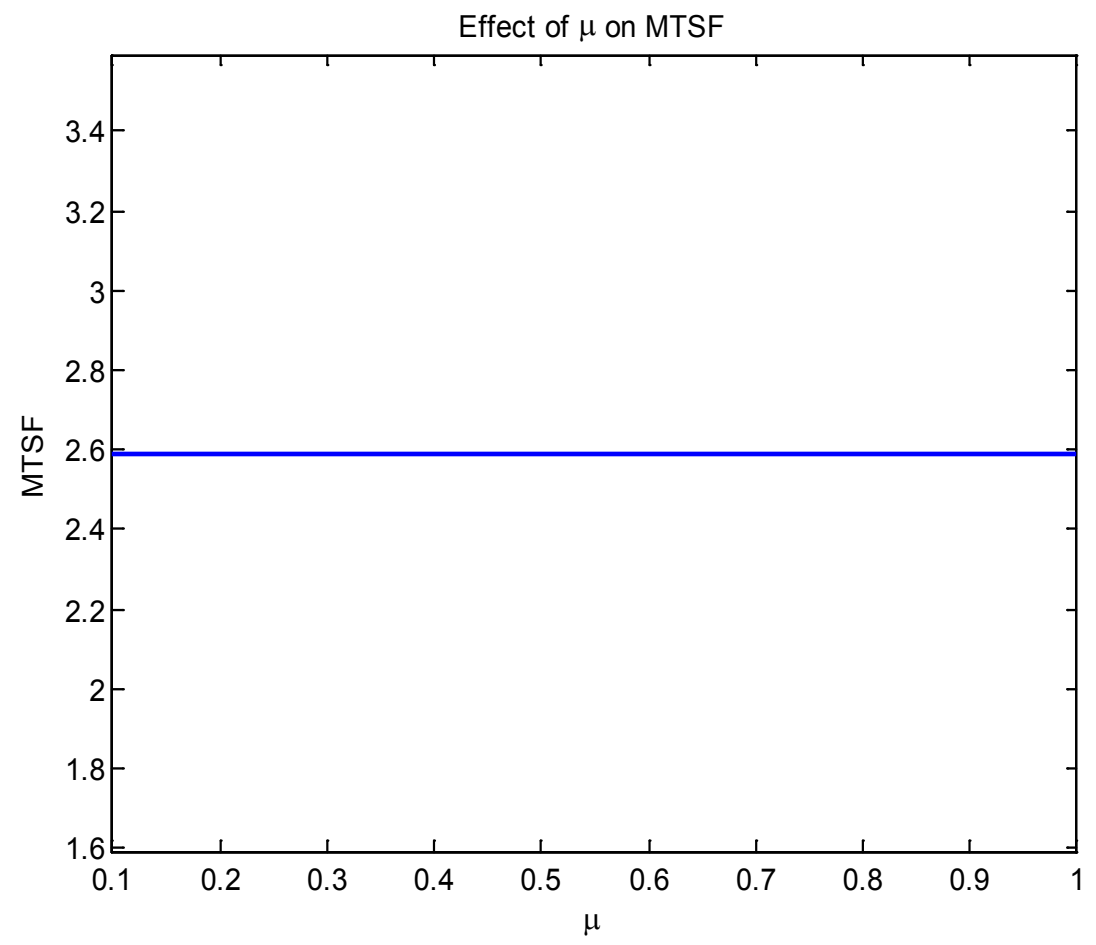

Figure 12. effect of $\mu$ on MTSF 


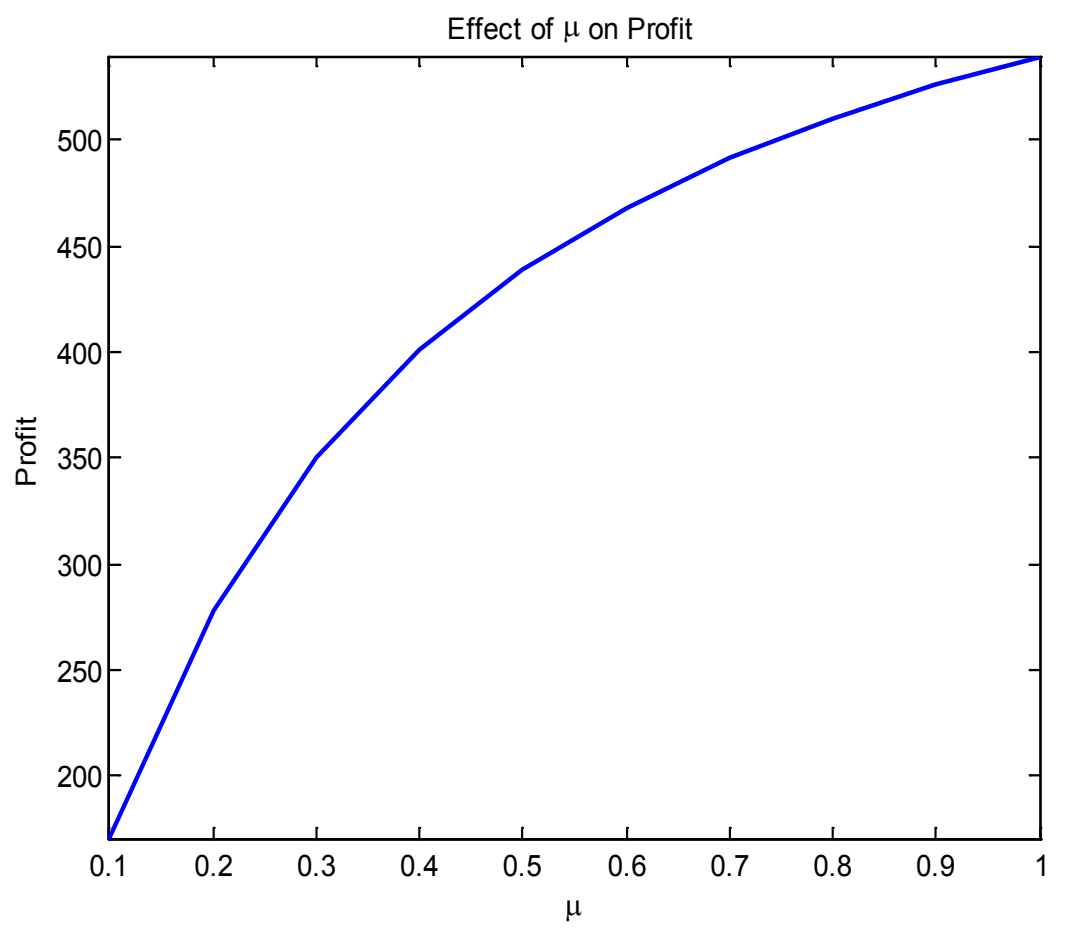

Figure 13. effect of $\mu$ on Profit

\section{Discussion}

Case I:

$$
\alpha_{2}=0.5, \quad \alpha_{3}=0.02, \quad \beta_{1}=0.5, \quad \beta_{2}=0.06 \text {, }
$$

$\beta_{3}=0.01, \lambda=0.6, \mu=0.5, C_{0}=1000, C_{1}=10$ and vary $\alpha_{1}$ for Figure 2 to 4 .

Case II:

$\alpha_{2}=0.01 \quad, \quad \alpha_{3}=0.02 \quad, \quad \alpha_{1}=0.01$

$\beta_{2}=0.06, \beta_{3}=0.9, \lambda=0.06, \quad \mu=0.05$,

$C_{0}=1500, C_{1}=10$ and vary $\beta_{1}$ for Figure 5 to 7 .

Case III:

$\alpha_{1}=0.09, \quad \alpha_{2}=0.5, \alpha_{3}=0.02, \quad \beta_{1}=0.05$,

$\beta_{2}=0.06, \quad \beta_{3}=0.01, \quad \mu=0.5, \quad C_{0}=1500$,

$C_{1}=10$ and vary $\lambda$ for Figure 8 to 10 .

Case IV:

$\alpha_{1}=0.5, \quad \alpha_{2}=0.5, \quad \alpha_{3}=0.02, \quad \beta_{1}=0.5$,

$\beta_{2}=0.06, \quad \beta_{3}=0.01, \lambda=0.6, C_{0}=1500$,

$C_{1}=10$ and vary $\mu$ for Figure 11 to 13 .

Figure 2 to 4 provides description of profit function, MTSF and system availability with respect to $\alpha_{1}$. From these figures, it is clear that both profit function, MTSF and system availability increase as $\alpha_{1}$ increases. In Figure 5 to 7 , the behavior of system availability, profit function and MTSF are shown with respect to $\beta_{1}$. It is observed that system availability decrease as $\beta_{1}$ increases. In Figure 8 to 10 , the behavior of system availability, MTSF and profit function with respect to $\lambda$. The results in these figures have shown that system availability, MTSF and profit function decrease as $\lambda$ increases. Figure 11 to 13 provides description of system availability, MTSF and profit function with respect to $\mu$. System availability and profit in Figure 11 and 13 increase with increase in $\mu$ while MTSF in Figure 12 is constant with respect to $\mu$.

\section{Conclusions}

In this paper, we developed the explicit expressions for the mean time to system failure (MTSF), system availability, busy period and profit function for the system and performed graphical study to see the behavior of failure rates and repair rates parameters on system performance. It is observed that from graphical study system performance increase with repair rates and decrease with failure rates.

\section{REFERENCES}

[1] Bhardwj, R.K. and Chander, S. (2007). Reliability and cost benefit analysis of 2-out-of-3 redundant system with general distribution of repair and waiting time. DIAS- Technology review- An Int. J. of business and IT. 4(1), 28-35

[2] Chander, S. and Bhardwaj, R.K. (2009). Reliability and economic analysis of 2-out-of-3 redundant system with priority to repair. African J. of Maths and comp. sci, 2(11), 230-236.

[3] Bhardwj,R.K., and S.C. Malik. (2010). MTSF and Cost effectiveness of 2-out-of-3 cold standby system with 
probability of repair and inspection. Int. J. of Eng. Sci. and Tech. 2(1), 5882-5889

[4] Taneja, G., A. Goyal and D.V. Singh (2011). Reliability and Cost benefit analysis of a system comprising one big unit and two small identical units with priority for operation/repair to big unit. Mathematical Sciences, Vol. 5 No. 3, 235-248

[5] Malik, S.C., Bharwaj, R.K. and Grewal, A.S. (2010) Probabilistic analysis of a system of two non identical parallel units with priority to repair to repair subject to inspection. Journal of reliability and statistical studies, vol. 3(1), pp 1-11

[6] Mahmoud, M.A.W. and Moshref, M.E. (2010). On a two unit cold standby system considering hardware, human error failures and preventive maintenance, Mathematics and Computer modeling, 51(5-6), pp 736-745.

[7] Yusuf, I. and Bala, S.I. (2012). Stochastic modeling of a two unit parallel system under two types of failures. International Journal of Latest trends in Mathematics, Vol. 2 (1), pp 44-53

[8] Kumar, J. and Kadyan, M.S. (2012). Profit analysis of a system of non identical units with degradation and replacement. International journal of computer application, vol. 40 (3), pp 19-25

[9] Sureria, J.K., Malik, S.C. and Anand, J. (2012). Cost benefit analysis of a computer system with priority to software replacement over hardware repair. Applied Mathematical
Sciences, vol. 6 (75), pp 3723-3734.

[10] El-Said, K.M.,. ( 2008).Cost analysis of a system with preventive maintenance by using Kolmogorov's forward equations method. Ame. J. of App. Sci. 5(4), 405-410

[11] Haggag, M.Y., (2009). Cost analysis of a system involving common cause failures and preventive maintenance.J. Maths. And Stat. 5(4), 305-310

[12] El-Said, K.M., and M.S. El-Sherbeny. ( 2005).Profit analy sis of a two unit cold standby system with preventive maintenance and random change in units. J. Maths and Stat.,1 (1) 71-77

[13] El-Said, K.M., and M.S. El-Sherbeny. (2005) .Evaluation of reliability and availability characteristics of two different systems by using linear first order differential equations. J. Maths and Stat.,1 (2) 119-123

[14] Wang, K., Hseih, C., and Liou, C. H. (2006). Cost benefit analysis of series systems with cold standby components and a repairable service station. Quality technology and quantitative management. Vol. 3(1), pp 77-92.

[15] Bhardwaj., R.K., and S.C. Malik (2010). MTSF and cost effectiveness of 2-out-of-3 cold standby system with probability of repair and inspection. International Journal of engineering, science and technology, vol. 2(10), 5882-5889 